\title{
Organisation of nucleosomal arrays reconstituted with repetitive African green monkey $\alpha$-satellite DNA as analysed by atomic force microscopy
}

\author{
Malte Bussiek • Gabriele Müller • \\ Waldemar Waldeck · Stephan Diekmann • \\ Jörg Langowski
}

Received: 30 November 2006/Revised: 5 April 2007/Accepted: 14 April 2007/Published online: 15 May 2007

(C) EBSA 2007

\begin{abstract}
Alpha-satellite DNA (AS) is part of centromeric DNA and could be relevant for centromeric chromatin structure: its repetitive character may generate a specifically ordered nucleosomal arrangement and thereby facilitate kinetochore protein binding and chromatin condensation. Although nucleosomal positioning on some satellite sequences had been shown, including AS from African green monkey (AGM), the sequence-dependent nucleosomal organisation of repetitive AS of this species has so far not been analysed. We therefore studied the positioning of reconstituted nucleosomes on AGM AS tandemly repeated DNA. Enzymatic analysis of nucleosome arrays formed on an AS heptamer as well as the localisation of mononucleosomes on an AS dimer by atomic force microscopy (AFM) showed one major positioning frame, in agreement with earlier results. The occupancy of this site was in the range of $45-50 \%$, in quite good agreement with published in vivo observations. AFM measurements of internucleosomal distances formed on the heptamer indicated that the nucleosomal arrangement is governed by sequence-specific DNA-histone interactions yielding defined internucleosomal distances, which,
\end{abstract}

\footnotetext{
M. Bussiek

Biophysical Engineering, Universiteit Twente, PO BOX 217, 7500AE Enschede, The Netherlands

G. Müller · W. Waldeck · J. Langowski ( $₫)$

Division of Biophysics of Macromolecules,

Deutsches Krebsforschungszentrum,

Im Neuenheimer Feld 580, TP3, 69120 Heidelberg, Germany

e-mail: j1@dkfz.de

S. Diekmann

Division of Molecular Biology, Fritz Lipmann Institut,

Beutenbergstraße 11, 07708 Jena, Germany
}

nevertheless, are not compatible with a uniform phasing of the nucleosomes with the AGM AS repeats.

Keywords Alpha-satellite DNA · Nucleosome arrays · Nucleosome positioning · Chromatin · Centromere . Green monkey · Atomic force microscopy $\cdot 5 \mathrm{~s}$ rDNA
Abbreviations
AS Alpha-satellite DNA
AGM African green monkey
AFM Atomic force microscopy
NCP Nucleosome core particle

\section{Introduction}

Satellite DNA consists of tandemly repeated sequences with monomer lengths of up to $500 \mathrm{bp}$ and copy numbers of up to millions. It is found in all eukaryotic species where it is concentrated in the centromeric regions of the chromosomes, as shown for example in humans (Schueler et al. 2001). The association of satellite DNA with essential processes ensuring genome stability, such as the assembly of the kinetochore (Willard 1998), is still unclear since these processes are not strictly sequence-dependent (Amor and Choo 2002). $\alpha$-satellite DNA (AS) might contribute to the formation of a centromere-specific chromatin structure. The presence of oligo-adenine tracts and intrinsic curvature are universal features of satellites, which therefore may have a role in positioning nucleosomes along DNA and in chromatin condensation (Fitzgerald et al. 1994). Indeed, sequence-dependent nucleosome positioning on satellite DNA fragments was observed in vitro (Linxweller and 
Horz 1985; Neubauer et al. 1986; Tanaka et al. 2005; Yoda et al. 1998). Also, satellite chromatin isolated from cell nuclei was found to differ from bulk chromatin by a higher compaction level which may be supported by the presence of a particularly ordered nucleosomal arrangement (Gilbert and Allan 2001).

Alpha-satellite DNA is characterised by a monomer length of $\sim 171 \mathrm{bp}$ and is primate specific. This DNA allows for a simple phase relationship between nucleosome binding and the repeat length. In fact, AS chromatin of African green monkey (AGM) (Cercopithecus aethiops) was used earlier to verify this phase relationship in vivo. The initial finding of a simple phase, based on micrococcal nuclease (MNase) digestions of cell nuclei (Brown et al. 1979; Musich et al. 1982), was challenged by the finding that the MNase cleaves the AGM AS non-randomly (Fittler and Zachau 1979; Horz et al. 1983). More detailed studies showed the occupation of different nucleosomal positions relative to the AS sequence in AGM-cells where, however, one highly preferred site consistently emerged in different approaches (Smith and Lieberman 1984; Wu et al. 1983; Zhang et al. 1983). Neubauer et al. (1986) identified nucleosomal positioning sites similar to those occurring in vivo by in vitro reconstitution experiments using $250 \mathrm{bp}$ AGM AS fragments. The major site was occupied by $80 \%$ of the nucleosomes on 250 bp DNA in vitro and by $35 \%$ in vivo (Neubauer et al. 1986; Zhang et al. 1983). These results argued for a strong contribution of sequencedependent DNA-histone interactions to the nucleosomal organisation in AS chromatin of this species, although nucleosomal positions and internucleosome spacing generally depend on many different factors in vivo. For instance, the sequence-dependent positioning signal contained in the 5S rDNA of Lytechinus variegatus can be overruled when nucleosomes are reconstituted in cell extracts (Blank and Becker 1996). Recently, Segal et al. (2006) found a high predicted occupancy of nucleosomes over centromeres indicating that centromere function requires enhanced stability of histone-DNA interactions that are encoded in the genomic sequence.

The importance of a correct nucleosomal positioning at centromeric chromatin is also indicated by the observation that the foundation centromeric protein CENP-B influences the position of nucleosomes along $\mathrm{AS}$ in vitro (Tanaka et al. 2005). CENP-B is the only known inner kinetochore protein to bind to a specific centromere sequence, the $17 \mathrm{bp}$ Cenp-B box, which occurs in a subset of $\alpha$-satellite monomers in humans (Masumoto et al. 1989). AS arrays of humans are composed of a number of divergent AS monomers organised into higher order repeat units, whereas arrays of AGM exhibit a simple and homogeneous monomer organisation and, furthermore, lack Cenp-Bboxes (Alexandrov et al. 2001; Goldberg et al. 1996).
Intriguingly, CENP-B was found to be absent from the centromeres of AGM although the protein is expressed (Goldberg et al. 1996). One interpretation for this result could be that CENP-B is dispensable in the centromeres of AGM, since the AS by itself efficiently defines nucleosomal positions (Goldberg et al. 1996), even though earlier the existence of a simple nucleosomal phase in vivo had been argued against, as mentioned above.

The motivation of the present work was to extend the earlier studies of nucleosome positioning on AS of this species. So far, the sequence-dependent assembly of nucleosome arrays has not been studied using repetitive AGM-AS, and nucleosomal positioning on DNA fragments containing the uninterrupted AGM-AS sequence has not been quantified. We therefore reconstituted oligonucleosome arrays with a heptamer and mononucleosomes with a dimer of the AGM AS and took advantage of atomic force microscopy (AFM) as single molecule approach to determine the nucleosomal organisation along the DNA. AFM can measure internucleosome distances with high precision (Allen et al. 1993; Mechelli et al. 2004; Yodh et al. 2002; Zlatanova et al. 1998) and also the quality of the reconstituted material can be characterised at the single molecule level. The height information, available through AFM imaging, gives molecular structural information (Nikova et al. 2004). Thus, the AFM approach circumvents drawbacks of enzymatic assays. Yet, we additionally used MNase and restriction enzyme digestion in order to find out whether chromatin reconstitution would give results similar to the earlier in vivo findings (Musich et al. 1982), taking into account that this approach may overestimate the extent of evenly spaced nucleosomes. Our AFM measurements of internucleosome distances show that the in vitro generated nucleosomal arrangement along the AGM AS heptamer is clearly defined by the sequence, yet deviates from a simple phasing. The preferred occupation of the known major positioning site was unambiguously detected by (1) MNase and EcoRI digestion and (2) by localising mononucleosomes reconstituted on the AGM AS dimer by AFM. The AS sequence repeat thus has a profound influence on the nucleosomal arrangement formed by reconstitution on this DNA and also the estimated occupancy of the major site was in a quite good agreement with the earlier in vivo findings.

\section{Material and methods}

\section{Preparation of DNA}

African green monkey $\alpha$-satellite DNA was prepared from CV1-cells, a permanent line derived from $C$. aethiops kidney cells. Whole genomic DNA was isolated, deproteinised and treated with EcoRI restriction endonuclease 
essentially as described (Gruss and Sauer 1975). The DNA samples were run on $1.0 \%$ agarose gels and stained with ethidium. EcoRI produces one large smear in the upper region of the gel and a number of discrete bands below, corresponding to multiples of the AS-monomer (Gruss and Sauer 1975). Our attempts to clone repetitive AGM AS embarked on two strategies. One was to directly excise EcoRI-fragments containing different repeat numbers from low melting agarose gels (FMC) and ligate these with the cloning vector pBluescript-IIKS+. Escherichia Coli XL10 Gold cells (Stratagene) were transformed with the ligation products and cloned. By this approach, vectors containing AS-monomers and AS-dimers but no larger repeat numbers could be successfully cloned. It was verified by DNAsequencing that the cloned DNA matches AGM AS: an isolated AS-dimer proved to be a tandem repeat of identical sequence. This yielded the plasmid pBluescript-IIKS- $\alpha 2$. The second approach to obtain longer AS-arrays was a stepwise self-ligation of the already available $\alpha 2$-insert. pBluescript-IIKS- $\alpha 2$ was subjected to a partial EcoRIdigestion and the cleavage products were separated on a $1.4 \%$ low melting agarose gel to isolate the $\alpha 2$-fragments. These were used for self-ligations at $4^{\circ} \mathrm{C}$ for $2-4$ days using $10 \mathrm{U} / \mu \mathrm{l}$ of T4 ligase. Afterwards, the DNA was ligated with pBluescript-KSII+ and we could obtain clones containing an AS-tetramer (pBluescript-KSII- $\alpha 4$ ). The $\alpha 4-$ insert was used for the next self-ligation step carried out accordingly. This resulted in a clone containing an ASheptamer (pBluescript-KSII- $\alpha 7$ ). DNA-sequencing was used to verify the tandem orientation of the AS-repeats.

A 208-6 construct was produced starting from the vector pPolI-5S-208-12 (a gift of Peter Becker) which contains a dodecamer of the $5 \mathrm{~S}$ rDNA nucleosome positioning sequence (Blank and Becker 1996; Simpson et al. 1985). This DNA was subjected to a partial digestion with AvaI to produce linearised vector-DNA with different repeat numbers of the 208-sequence. The cleaved vector was religated and cloned. A clone containing a hexamer of the 208 -sequence was identified by $1.2 \%$ agarose gel electrophoresis after cleavage of the purified vector with PstI.

To prepare the target DNA-fragments for the chromatin reconstitution experiments, bacteria were grown in Luria Bertani medium at $37^{\circ} \mathrm{C}$ for $24 \mathrm{~h}$ and the cells were harvested by centrifugation at $6,000 \mathrm{rpm}$ for $20 \mathrm{~min}$ at $4^{\circ} \mathrm{C}$. The plasmids were isolated using the Nucleobond-kit (Machery-Nagel). AS-vectors were cleaved with NotI and XhoI to separate a fragment containing the AS-insert flanked on both sides by 33 and $37 \mathrm{bp}$, respectively (172$\alpha 2,415$ bp and 172- $\alpha 7,1,275 \mathrm{bp})$. The 208-6 DNA was separated from pPolI-5S-208-6 by cleavage with PstI and $X b a I$. The cleavage products were run on $1.4 \%$ low melting agarose gels, the target fragments were excised from the gels and purified using the gel purification-kit (Qiagen).

\section{Chromatin reconstitution}

Recombinant histone proteins of Xenopus laevis were overexpressed and purified as described (Kepert et al. 2003; Luger et al. 1999). Histone octamers were formed by incubation of a stoichiometric mix of the histones for $30 \mathrm{~min}$ in unfolding buffer (7 M Urea, $20 \mathrm{mM}$ Tris- $\mathrm{HCl}$, $\mathrm{pH}$ 7.4) and subsequent dialysis against refolding buffer (20 mM Tris-HCl, $\mathrm{pH} 7.4 ; 0.1 \mathrm{mM}$ EDTA; $0.5 \mathrm{mM} \beta$ mercaptoethanol; $2 \mathrm{M} \mathrm{NaCl}$ ). An electrophoretic analysis of the used octamer preparation is shown elsewhere (Bussiek et al. 2005).

Nucleosome arrays with 172- $\alpha 7$ and mononucleosomes with $172-\alpha 2$ were reconstituted by the salt dialysis method. Octamers were mixed with the DNA in $10 \mathrm{mM}$ Tris- $\mathrm{HCl}$, $\mathrm{pH} 7.5 ; 2 \mathrm{M} \mathrm{NaCl}$ and kept at $30^{\circ} \mathrm{C}$ for $30 \mathrm{~min}$. The mixture was transferred to mini dialysis tubes (Pierce, Rockford, USA) and dialysed against buffers with stepwise decreasing $\mathrm{NaCl}$ concentrations at $4^{\circ} \mathrm{C}(1.8,1.4,1.0,0.8$, $0.6,0.4,0.2$ and $0.1 \mathrm{M}$ for $1 \mathrm{~h}$ each and $0.005 \mathrm{M}$ over night). The reconstitutions took place at different weight ratios of histone-octamer to DNA, ranging between values of 0.3-1.0. The DNA mass was $0.02 \mu \mathrm{g} / \mu \mathrm{l}$.

Analytical digestions with micrococcal nuclease and EcoRI restriction endonuclease

172- $\alpha 7$ DNA, either naked or reconstituted with histones, was digested with micrococcal nuclease (MNase). Reactions took place in $10 \mathrm{mM}$ Tris- $\mathrm{HCl}$, pH 7.5; $5 \mathrm{mM} \mathrm{NaCl}$; $0.5 \mathrm{mM} \mathrm{CaCl}_{2}$ using $0.3 \mathrm{mU} / \mu \mathrm{l}$ MNase and $0.02 \mu \mathrm{g} / \mu \mathrm{l}$ of the DNA. The reactions were stopped by mixing $50 \mu \mathrm{l}$ aliquotes of the reaction with $5 \mathrm{mM}$ EDTA after varying reaction times. Then, the DNA was isolated with phenol/ chloroform/isoamylalcohol, precipitated with ethanol and resuspended in $15 \mu \mathrm{l}$ Tris- $\mathrm{HCl}$, $\mathrm{pH}$ 7.5, $0.1 \mathrm{mM}$ EDTA. The DNA was analysed on $1.2 \%$ agarose gels run in $1 \times$ TBE-buffer using ethidium staining.

For analytical digestion with EcoRI, another fraction of the reconstituted chromatin was first digested with MNase to produce mononucleosomal DNA. The reaction conditions and purification of the DNA was carried out as described above, where a total of $1.5 \mu \mathrm{g}$ of DNA were digested. An aliquot corresponding to $1.0 \mu \mathrm{g}$ of the purified DNA was subjected to EcoRI digestion for $2 \mathrm{~h}$. The two DNA-samples (MNase and MNase $+E c o$ RI) were run on a $1.7 \%$ agarose gel in $1 \times \mathrm{TBE}$, together with a 20 bp-ladder and stained with ethidium.

Atomic force microscopy

Atomic force microscopy was performed in air and in solution using a Multimode ${ }^{\mathrm{TM}}$ (Digital Instruments) oper- 
ated in tapping mode essentially as described (Bussiek et al. 2005). Mica modified with poly-L-lysine (PL) served as support for sample immobilisation. Freshly cleaved mica was pre-treated with $30 \mu \mathrm{l}$ of an aqueous solution of PL (Sigma) at a concentration of $10 \mu \mathrm{g} / \mathrm{ml}$. After incubation for $30 \mathrm{~s}$, unbound PL was removed by rinsing the mica disc with $4.0 \mathrm{ml}$ of Millipore purified water followed by drying under a nitrogen stream. The chromatin was diluted to concentrations ranging between 0.5 and $2.0 \mathrm{nM}$ (depending on the DNA size) in $10 \mathrm{mM}$ Hepes-NaOH, pH 8.0, supplemented with salt as indicated in Sect. "Results", Thirty microlitres of the dissolved chromatin solution were placed on the PL-mica. For scanning directly in the adsorption buffer, silicon nitride probes (type NP-S20, Veeco Instruments) were used at drive frequencies of $8.0-9.5 \mathrm{kHz}$ and a set point of $0.3-0.4 \mathrm{~V}$. For scanning in air, the mica disc was washed carefully with $2.0 \mathrm{ml}$ of Millipore water after incubating with the chromatin solution for $1 \mathrm{~min}$. Then, the disc was dried with nitrogen and subsequently scanned using etched silicon probes (type $\mathrm{NCH}$, Nanosensors) at drive frequencies of $280-320 \mathrm{kHz}$ and set point of $2.0-$ $2.2 \mathrm{~V}$. The images were recorded both in solution and in air at a scan diameter of $2 \times 2 \mu \mathrm{m}$, a scan rate of $1-2 \mathrm{~Hz}$ and a resolution of $512 \times 512$ pixels.

Image analysis

Atomic force microscopy images were flattened and zooms of individual complexes were produced using the Nanoscope IIIa software (version 5.12r3, Veeco Instruments).

Measurements of nucleosomal heights were done with the section analysis tool available in the same software package. This was also used to decide in some critical cases, whether a pair of two closely localised nucleosomes or a single one was present. When the cross-section showed two peaks or clearly a plateau next to a peak, two closely localised nucleosomes were assumed.

Length measurements along DNA or chromatin contours were done using the freehand line tool in the program ImageJ (version 3.13, National Institute of Health). To measure the lengths of DNA fragments complexed into nucleosomes $\left(L_{\text {complex }}\right)$, contours were traced along the entry and exit sites of the DNA strands into the nucleosomes. The nucleosome diameter measured by AFM is usually larger than the real diameter due to tip convolution, which means that the actual entry/exit sites cannot be unambiguously localised. To account for this error, the apparent entry/exit sites were always connected through the nucleosomal centres. In this way, the length of free DNA outside the nucleosomes $\left(L_{\text {free}}\right.$; free linker DNA and DNA termini) could be estimated by subtracting the known diameter of the nucleosome core particle (NCP) of $11 \mathrm{~nm}$ :
$L_{\text {free }}=L_{\text {complex }}-n_{\text {ncp }} \cdot 11 \mathrm{~nm}$,

where $n_{\text {ncp }}$ is the number of NCPs per complex.

Internucleosomal spacings between consecutive nucleosomes in the arrays were measured as centre-to-centre (cc) distances. The $c c$-distances were measured along DNA, when two nucleosomes were separated by a distance sufficiently long to make the intervening DNA visible. Otherwise, when two nucleosomes appeared close to one another, a straight line between the nucleosomal centres was assumed. The resolution limit that allows distinguishing nearby nucleosomes closely coincides with the NCP diameter. Since a correlation between the $c c$-distance and the internucleosomal separation along the DNA is not justified in this range of small distances, a minimum value of $11 \mathrm{~nm}$ was defined for the data analysis. The accuracy of the measurement as given by the pixel-size of the zooms used here is $0.5 \mathrm{~nm}$. Pairs of nucleosomes had to fulfil some criteria in order to be included in the $c c$-distance measurements: it had to be unambiguous that the nucleosomes are ordered one after another along the DNA and the nucleosomes had to feature a certain minimal height (see below). Finally, nucleosomes localised directly at DNA termini were excluded from the $c c$-distance measurements.

\section{Results}

We studied the positioning of reconstituted nucleosomes on AGM $\alpha$-satellite (AS) tandemly repeated DNA.

\section{The cloned AGM AS}

We constructed vectors containing AGM AS-DNA monomers as tandem repeats of up to seven. Figure 1a shows the DNA-sequence of the isolated AS (Fig. 1a, 1) aligned with the sequence (Figs. 1a, 2) analysed earlier in nucleosome reconstitution experiments (Neubauer et al. 1986). This sequence did not contain the HindIII site that is present in the major fraction of AGM AS (Musich et al. 1982; Neubauer et al. 1986; Wu et al. 1983). The vectors containing the AS-fragments used for the reconstitution experiments (172- $\alpha 2$ and 172- $\alpha 7$ ) were NotI/XhoI digested and analysed on the agarose gel in Fig. 1b. For the reconstitution experiments, the target fragments were isolated from the agarose gels.

DNA-sequencing verified the tandem orientation of the repetitive AS-monomers. Interestingly, all sequenced constructs so far obtained by self-ligation of AS-dimers or of AS-tetramers, contained tandemly oriented repeats (a total of 12 clones with various repeat numbers were sequenced), potentially due to an intolerance of the bacteria 

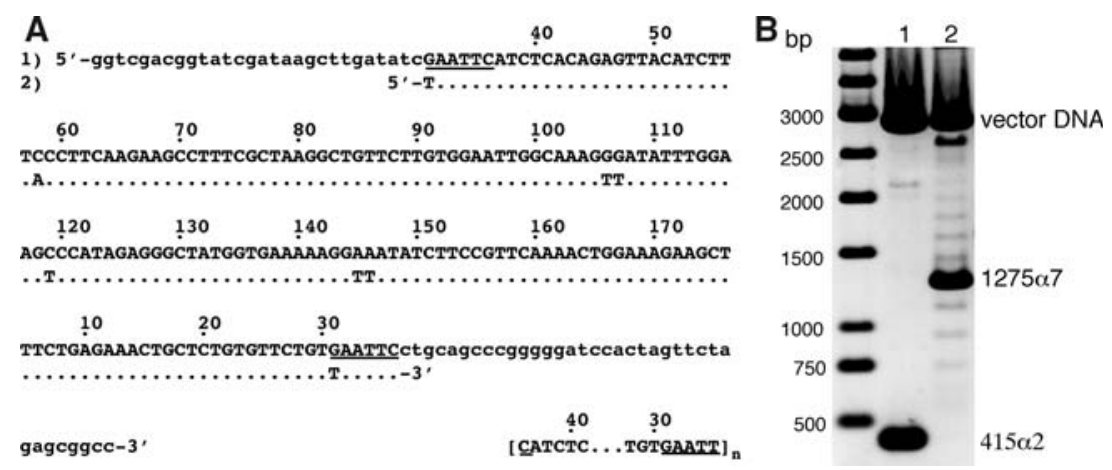

Fig. 1 Characterisation of the satellite DNA fragments used for chromatin reconstitutions. a The cloned AGM AS (1) is shown in upper case letters and aligned with the sequence (2) analysed earlier in reconstitutions experiments [adapted from Neubauer et al. 1986]. Lower case letters show flanking vector DNA. The EcoRI recognition sites are underlined. Numbering of sequence positions is according to Rosenberg et al. (1978). The sequence in brackets indicates the repetition of the $172 \mathrm{bp}$ AS monomer starting and ending at the

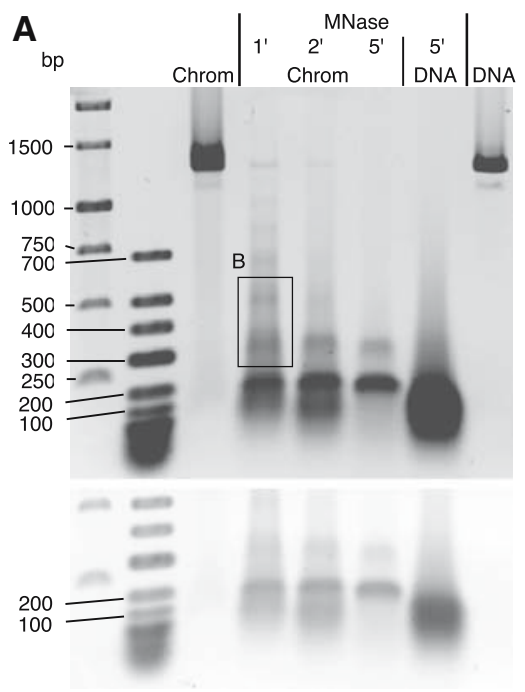

Fig. 2 Micrococcal nuclease (MNase) and EcoRI cleavage of reconstituted 172- $\alpha 7$ nucleosome arrays. a Arrays (chrom) were treated with MNase for 1-5 min. Samples were loaded on the $1.2 \%$ agarose gel together with untreated arrays and naked 172- $\alpha 7$ DNA, also either treated with MNase for $5 \mathrm{~min}$ or untreated. Untreated arrays and naked DNA migrate similarly in the agarose gel (DNA compaction and the increase in molecular weight by complexing with histones approximately compensate). First two lanes marker DNA. A photograph of the gel taken at higher light intensity is included

to inverted repeats. The formation of odd or even numbers of repeated monomers after self-ligation indicates that entire AS-monomer units can be excised during bacterial growth. The gel in Fig. 1b also demonstrates that bacterial cell lines can contain a distribution of different AS repeat numbers centred around one prevailing length. Growing
EcoRI site in our case ( $n=2,172-\alpha 2$ DNA; $n=7,172-\alpha 7$ DNA). b Agarose-gel electrophoresis of the AS fragments after separation from the cloning vector with NotI and XhoI (lane $1172-\alpha 2,415$ bp, lane 2 $172-\alpha 7,1,275 \mathrm{bp})$. Note, that the target fragments were isolated from agarose gels for the reconstitution experiments. Here, the NotI/XhoI digestion was directly loaded on the gel to also show the occurrence of a distribution of different AS repeat numbers around the major repeat length of $n=7$ in the cell line containing pBluescript-KSII- $\alpha 7$

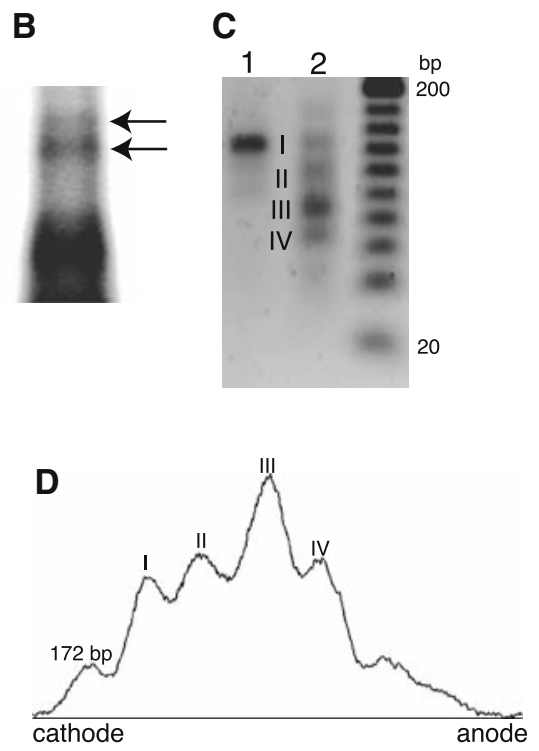

underneath. b Magnified area of the same gel, marked by the frame in a. Arrows are shown to suggest a biphasic fragment pattern produced by the MNase. c An aliquot of the same arrays was digested with MNase for 7 min to obtain pure core particle length DNA. An aliquot of the cleavage product was digested with EcoRI and both samples were loaded on the $1.7 \%$ agarose gel (lane 1 core particle DNA, lane 2 EcoRI digestion) together with a 20 bp ladder. Both gels were stained with ethidium. d Densitometric trace of lane 2 in $\mathbf{c}$ calculated with NIHimage software (version 1.63) after background subtraction

the bacteria for several days showed the stable maintenance of this distribution as well as the prevailing repeat number (data not shown). Preliminary results show that this distribution can be also used to generate successively longer repeats by isolating the largest fragments from gels (not shown). 
Gel electrophoretic characterisation of chromatin reconstituted with $172-\alpha 7$

A proper reconstitution of nucleosomes as well as a regular nucleosomal periodicity can be identified by a digestion with MNase. The cloned AS heptamer $(172-\alpha 7)$ was reconstituted with histones at an octamer to DNA weight ratio of 1.0. The chromatin was treated with MNase for three different periods of time, deproteinised and electrophoresed. Figure 2a shows the expected protection of the nucleosomal core particle DNA, which is not observed when naked DNA was digested. Larger fragments were observed with lengths that are multiples of the AS-monomer length, suggesting the existence of regularly spaced nucleosomes. This ladder successively disappears with digestion time. On the other hand, a sequence-specific cleavage of the AGM AS may likewise have produced the ladder (Horz et al. 1983). The magnified area of the gel (Fig. 2b) indeed suggested the presence of two phases and also a considerable smear superimposed the bands. In order to analyse nucleosomal binding further, pure core particle length DNA produced by MNase cleavage of the same reconstituted material was digested with EcoRI restriction endonuclease. This enzyme was chosen on the basis of earlier results that have localised the EcoRI cleavage site near the centre of a major nucleosomal positioning frame in vivo and in vitro (Musich et al. 1982; Neubauer et al. 1986; Zhang et al. 1983). The agarose gel in Fig. 2c and the densitometric trace in Fig. 2d demonstrate that EcoRI has produced discrete bands, indicating that the nucleosomes have occupied preferred sites along the 172- $\alpha 7$ DNA. The largest fragment (Fig. 2c, I) corresponds to core particle length (uncleaved) DNA, which must have originated from nucleosomes formed on one or different positions that do not incorporate the EcoRI site. Additionally, three smaller fragments were produced, one of which with size $\sim 85$ bp prevailed (Fig. 2c, III). The size $\sim 65$ bp of another fragment (IV), when added to the $85 \mathrm{bp}$, approximately yields core particle length DNA. We therefore assume that these two fragments were produced by cleavage of the same nucleosomal core DNA. In support of this, the intensities of these two bands (Fig. 2d) differ by a factor of 1.5 , which roughly would be expected based on the molecular weight difference (factor of 1.3, assuming the 85 and $65 \mathrm{bp}$ ). Thus, the appearance of these fragments indicates a preferred nucleosomal binding frame, whose centre is located about 10 bp away from the EcoRI site, in good agreement with the earlier results (Musich et al. 1982; Neubauer et al. 1986; Zhang et al. 1983). Next, the gel indicates a second weaker positioning site by the presence of a $\sim 120 \mathrm{bp}$ fragment (Fig. 2c, II). Here, however, the corresponding smaller fragment (expected size $27 \mathrm{bp}$ to yield core particle length DNA) was faint. Based on the densitometric analysis, $50 \%$ of the nucleosomes were bound at the most preferred site. Note that the major site occurs six times in the 172- $\alpha 7$ DNA and is interrupted at the fragment ends (since, in our case, monomers start and end at the EcoRI site).

\section{AFM imaging of $172-\alpha 7$ nucleosome arrays}

The gel results support an at least partially regular nucleosomal organisation on the 172- $\alpha 7$ template. It remains unclear, however, to what extent a fraction of the nucleosomes were positioned randomly. Therefore, AFM was used to determine distances between consecutive nucleosomes assembled along the 172- $\alpha 7$ AS heptamer DNA. As a control, nucleosome arrays were reconstituted on a hexamer repeat of $2085 \mathrm{~S}$ rDNA to obtain a reference with well-documented properties. Tandem repeats of a $208 \mathrm{bp}$ long DNA containing $5 \mathrm{~S}$ rDNA of $L$. variegatus are often used as a model system for evenly spaced nucleosome arrays (Carruthers et al. 1998; Simpson et al. 1985) and their distribution of internucleosomal distances had been determined by AFM (Allen et al. 1993; Mechelli et al. 2004; Nikova et al. 2004; Yodh et al. 2002).

Scanning was done on air dried samples which ensured that all nucleosomes in the arrays were clearly distinguishable. The chromatin was immobilised at conditions that trap DNA on the surface irreversibly (Bussiek et al. 2003). We believe (and provide evidence) that this prevents the sample from extensive structural distortions once bound. Figure $3 \mathrm{a}$ gives an example of the scans obtained and Fig. 3b-i shows zooms of individual nucleosome arrays reconstituted with 172- $\alpha 7$ (B-G) and with 208-6 DNA $(\mathrm{H}, \mathrm{I})$. Adsorption of the arrays to the surface under low salt conditions yielded the expected extended conformations (Fig. 3b-e; H and I) and more compact conformations were observed in the presence of $\mathrm{MgCl}_{2}$ (Fig. 3f, g). Visual inspection suggested spaced nucleosomes on both sequences, although frequently nucleosomes appeared closely stacked on each other (arrows in Fig. 3). Height measurements of those particles identified as nucleosomes yielded $2.6( \pm 0.7 \mathrm{SD}) \mathrm{nm}$, in agreement with previous measurements under the same scanning conditions in air (Bussiek et al. 2005). All particles with heights below $1.9 \mathrm{~nm}$ were rejected in order to ensure that only properly folded nucleosomes were considered in the data analysis. The DNA was not fully saturated with nucleosomes since we expected to identify 'gaps' corresponding to unoccupied repeats, if preferred nucleosomal binding sites existed. For comparison, arrays were also scanned in liquid, which yielded the same extended shapes in low salt buffer (not shown). A quantitative analysis after scanning in liquid was, however, not useful because nucleosomes and DNA were not imaged with the required sharpness. On contrary, 

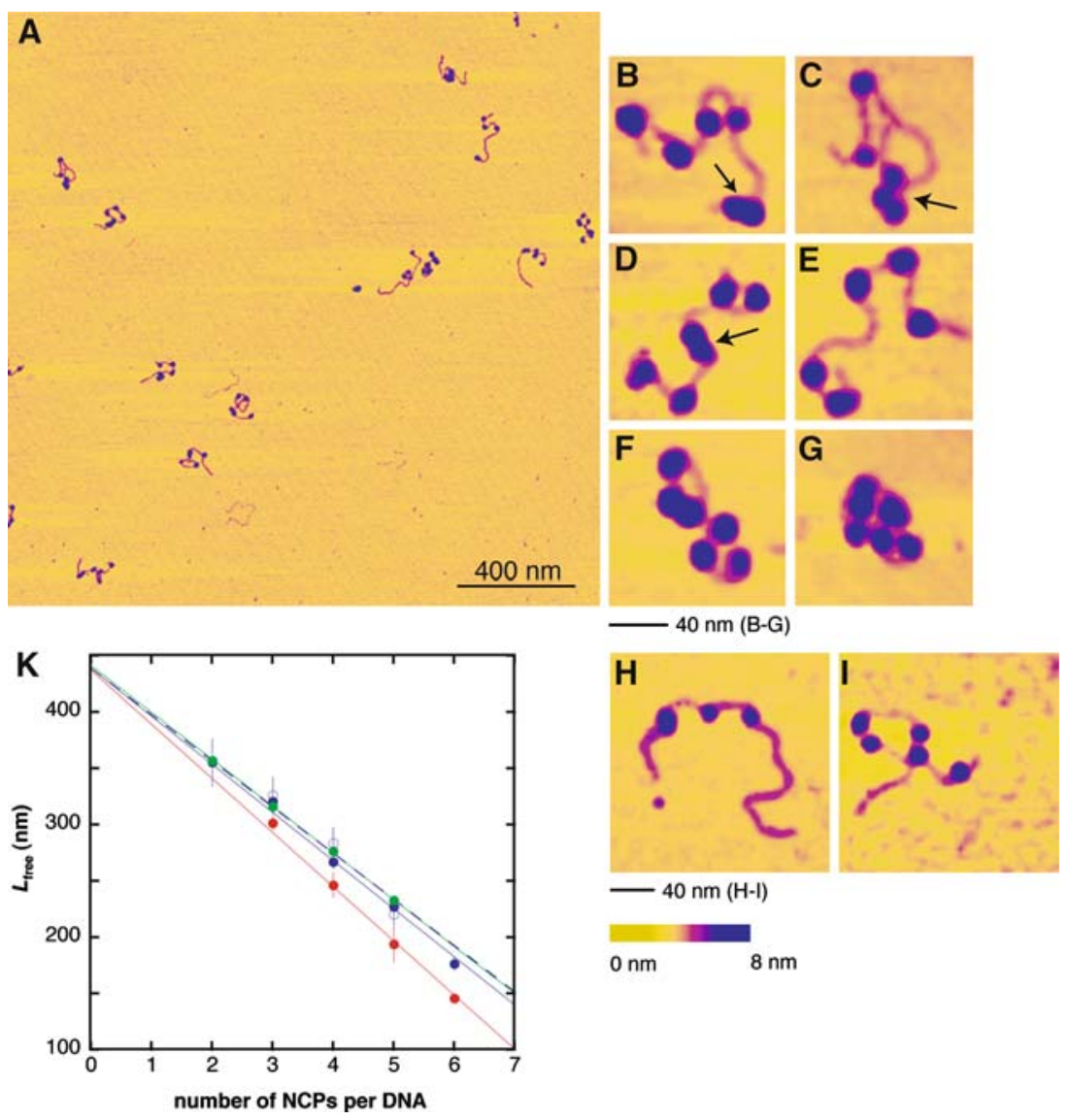

Fig. 3 a-i Atomic force microscopy imaging in air of nucleosome arrays. The chromatin was reconstituted at a nominal octamer to DNA weight ratio of 0.5 and adsorbed onto the surface in Hepes- $\mathrm{NaOH}$ buffer (10 mM, pH 8.0). a Overview scan of 172- $\alpha 7$ arrays adsorbed in the buffer additionally containing $10 \mathrm{mM} \mathrm{NaCl}$. b-g Zooms of individual 172- $\alpha 7$ arrays [adsorption buffer $+10 \mathrm{mM} \mathrm{NaCl}(\mathbf{b}, \mathbf{c})$, no additional salt $(\mathbf{d}, \mathbf{e})$ and $10 \mathrm{mM} \mathrm{NaCl}, 2 \mathrm{mM} \mathrm{MgCl} 2$ (f, g)]. The arrows mark nucleosomes in a close contact. $\mathbf{h}$, i Examples of 208-6

scanning of mononucleosomes in liquid yielded high quality images, as shown below. We explain this difference by a more unstable scanning process of nucleosome arrays, because a local conglomeration of nucleosomes has the effect of a locally higher surface roughness.

Internucleosomal distances were measured in a way that allowed a direct comparison with the expected nucleosomal repeat lengths of 172 and $208 \mathrm{bp}$. This was accomplished by determining the wrapped nucleosomal DNA length for the given experimental conditions. Low salt concentrations during the surface adsorption were selected so that nucleosomes formed extended conformations. As a consequence, linker DNAs could be well identified allowing for precise length measurements. However, it has to be taken into account that, due to repulsive electrostatic forces under low salt conditions, core particle DNA may partially unwrap from the histone surface to become linker DNA (Hamiche et al. 1996), a phenomenon that we also

nucleosome arrays adsorbed in buffer $+10 \mathrm{mM} \mathrm{NaCl}$. $\mathbf{k}$ Linear compaction of the DNA with number of NCPs per array $\left(n_{\text {ncp }}\right)$. Blue, closed symbols, continuous line indicate 172- $\alpha 7$ arrays in buffer $+10 \mathrm{mM} \mathrm{NaCl}$, blue, open symbols, dashed line indicate $172-\alpha 7$ arrays in buffer without additional $\mathrm{NaCl}$, green indicates 2086 arrays in buffer $+10 \mathrm{mM} \mathrm{NaCl}$, red indicates $172-\alpha 7$ arrays in buffer $+10 \mathrm{mM} \mathrm{NaCl}, 2 \mathrm{mM} \mathrm{MgCl}_{2}$. Error bars correspond to standard errors

observed under the present experimental conditions (Bussiek et al. 2005). We therefore estimated the average length of wrapped DNA $\left(L_{\mathrm{ncp}}\right)$ from the linear compaction of the DNA by complexing into nucleosomes. The $172-\alpha 7$ and the 208-6 nucleosome arrays were deposited onto the mica surface under the same low salt conditions (10 mM Hepes$\mathrm{NaOH}, \mathrm{pH} 8.0,10 \mathrm{mM} \mathrm{NaCl}$ ). All arrays with an unambiguous number and order of nucleosomes were included in these length measurements (a total of 106). Note that Fig. $3 \mathrm{f}$ and $\mathrm{g}$ give examples of excluded arrays. These are shown to demonstrate the expected compaction in the presence of $\mathrm{Mg}^{2+}$. Figure 3k shows that the nucleosome arrays formed on the DNA templates indeed shortened linearly with the number of nucleosomes $n_{\text {ncp. }} L_{\text {free }}$ measured as described in Sect. "Materials and methods" rather than the entire array length was plotted versus $n_{\text {ncp }}$ to be able to include the length of the naked DNA fragments as data points for the calculation of linear regressions 
(433 nm for 172- $\alpha 7$ DNA and $442 \mathrm{~nm}$ for 208-6 DNA). The slopes of the calculated regression lines, giving the wrapped DNA length, were about identical for the two nucleosomal arrays and removal of the $\mathrm{NaCl}$ from the buffer of one other sample had no effect, thus all three values obtained under these low salt conditions were averaged. This yielded a wrapped DNA length $L_{\text {ncp }}=$ $42 \mathrm{~nm}$ (or $123 \mathrm{bp}$ for $0.34 \mathrm{~nm} / \mathrm{bp}$ ), significantly less than the known core particle length of $147 \mathrm{bp}$. A wrapped DNA length of $\sim 120$ bp could be confirmed independently by measurements of mononucleosomes (see below). To further control the measurement accuracy, the wrapped DNA length was determined for a sample prepared in the presence of $\mathrm{MgCl}_{2}$, which yielded $48 \mathrm{~nm}$ (or $141 \mathrm{bp}$ ), very close to the core particle length.

Next we determined the distribution of the centre-tocentre $(c c)$ internucleosomal distances (Fig. 4). The data reveal a non-random nucleosomal positioning along the $172-\alpha 7$ DNA. This is indicated by a clearly separated initial main peak followed by a recurring preference for a larger distance, consistent with the existence of preferred binding sites, a fraction of which was unoccupied (Yodh et al. 2002). The distribution includes three different samples with a combined average number of nucleosomes $n_{\text {ncp-av }}=$ 4.2. Next, the $c c$-distances were plotted for only those complexes carrying less than five nucleosomes to obtain a subset sample with more unoccupied binding sites. Figure $4 \mathrm{~b}$ shows that the first recurring peak now is more accentuated and sharper compared to the distribution in Fig. 4a. Even a second and a third recurring peak are suggested, but not statistically secure.

For comparison, nucleosome arrays were reconstituted with 208-6 DNA, expected to produce defined regular nucleosome arrays due to positioning in the 5S rDNA sequence (Carruthers et al. 1998; Meersseman et al. 1991; Yodh et al. 2002) and thus also serving as a control for appropriate experimental conditions. The reconstitution with the 208-6 DNA yielded an average number of nucleosomes that exactly matched the subset sample in Fig. $4 \mathrm{~b}\left(n_{\text {ncp-av }}=3.2\right)$. Figure $4 \mathrm{c}$ shows a sharply defined preference for $c c$-distances around the third bin (35$47 \mathrm{~nm}$ ) for this sample. The peak, as also seen in Fig. 4a, is differentiated from a recurring peak and additionally from a distinguishable preference for very small $c c$-distances (those found in the first bin). These correspond to the many contacting nucleosome pairs. Close contacts in the 172- $\alpha 7$ arrays are as well reflected in a high number of counts in the first bin in Fig. 4a although not as a separated peak. Examples can be seen in Fig. 3b-d. Importantly, positioning in the $5 \mathrm{~S}$ rDNA led to a sharp initial peak, consistent with other reported AFM data (Mechelli et al. 2004; Nikova et al. 2004; Yodh et al. 2002).
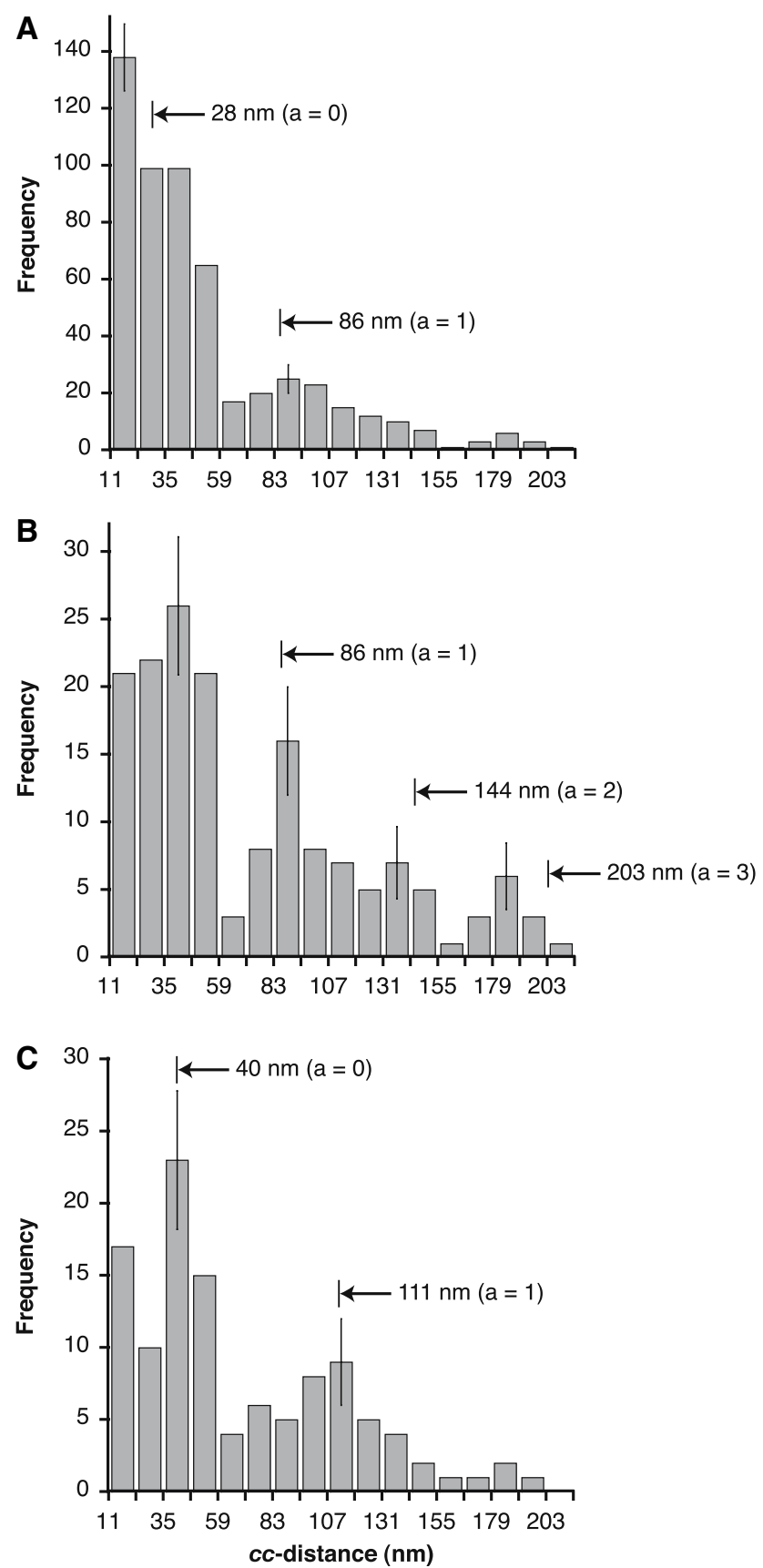

On the basis of the average wrapped DNA length $\left(L_{\text {ncp }}\right.$ $\sim 120 \mathrm{bp}$ ), expected $c c$-distances $\left(c c_{\text {exp }}\right)$ for monomer lengths $\left(L_{\text {mono }}\right)$ of 172 and $208 \mathrm{bp}(58$ and $71 \mathrm{~nm}$, respectively) can be calculated assuming that

$c c_{\text {exp }}=L_{\mathrm{mono}}-L_{\mathrm{ncp}}+11 \mathrm{~nm}+\left(a \cdot L_{\mathrm{mono}}\right), a=0,1,2 \ldots$

where $a$ is the number of unoccupied repeats between successive nucleosomes. These distances and their positions along the $x$-axes are indicated by numbers and 
Fig. 4 Distributions of $c c$-distances in 172- $\alpha 7$ and 208-6 nucleosome arrays, measured by AFM after adsorption under low salt conditions. a $c c$-distances in 172- $\alpha 7$ arrays measured in three samples with a combined average number of NCPs per array $n_{\text {ncp-av }}=4.2$. b $c c$ distances for exclusively 172- $\alpha 7$ nucleosome arrays with number of NCPs $n_{\text {ncp }}<5$, corresponding to $n_{\text {ncp-av }}=3.2$. c $c c$-distances measured in 208-6 nucleosome arrays $\left(n_{\mathrm{ncp}-\mathrm{av}}=3.2\right)$. Numbers and arrow heads indicate $c c$-distances expected for a uniform nucleosomal phasing with the monomer lengths of $172 \mathrm{bp}$ (a, b) and $208 \mathrm{bp}$ (c), as calculated assuming Eq. (2). $a$ is the number of unoccupied repeats between successive nucleosomes. The distance distribution begins at a value of $11 \mathrm{~nm}$, the diameter of the NCP, meaning that a few measured distance values smaller than $11 \mathrm{~nm}$ were added to the first bin. The bin width of $12 \mathrm{~nm}$ corresponds to the length difference between monomer lengths of 172 and 208 bp (36 bp). Error bars indicate the square root of the number of observations in each bin

arrowheads in Fig. 4, indicating a good agreement with measured (and assignable) peak maxima. In the case of a uniform phasing along the AGM AS repeats, the initial peak for the 172- $\alpha 7$ nucleosome arrays should be centred at about $30 \mathrm{~nm}$ (in the second bin) and not exceed values of $\sim 40 \mathrm{~nm}$, taking into account that variation in the wrapped DNA length likely broadens the $c c$-distance distribution. For an estimate of this variation we can assume a standard deviation of $\pm 28 \mathrm{bp}$ (corresponding to $\pm 9 \mathrm{~nm}$ ), as determined in 172- $\alpha 2$ mononucleosomes (see below). The observed distances clearly exceed this boundary and actually show a high occurrence of internucleosomal separations of up to three times that expected for uniformly phased nucleosomes. This is different in the 208-6 nucleosome arrays: here, the width of the initial main peak suggests that most nucleosomes are bound at or near the same positioning site in the $5 \mathrm{~S}$ rDNA repeats (neglecting close contacts). It is known for the the 5S rDNA that alternative positioning sites around the main site exist (e.g. Meersseman et al. 1991). The comparison of both sequences shows that multiple positioning in the 172- $\alpha 7$ DNA leads to a higher variation between internucleosomal spacings, which can be due to a higher proportion of nucleosomes positioned out of the main phase and by larger distances between the alternative sites. Apart from this observation, the clear separation of the main and first recurring peaks observed for both sequences indicates clearly the strong sequence-dependence of the nucleosome array formation on repetitive AGM-AS.

\section{AFM imaging of 172- $\alpha 2$ mononucleosomes}

To further quantify the nucleosomal positioning along the AGM AS, mononucleosomes were reconstituted with the AS dimer containing 172- $\alpha 2$ DNA (415 bp). Here, the complexes were imaged in liquid (10 mM Hepes- $\mathrm{NaOH}$, $\mathrm{pH} 8.0,10 \mathrm{mM} \mathrm{NaCl}$ ) and the mica surface was modified with the same amount of polylysine that was used for immobilising the nucleosome arrays to ensure constant adsorption conditions. Figure 5a shows a representative overview image displaying different kinds of complexes: mononucleosomes, a few dinucleosomes and uncomplexed DNA. Figure 5b-e shows zooms of individual complexes with different nucleosomal positions along the 172- $\alpha 2$ fragment.

We first characterised the heights of the mononucleosomes. The height values were distributed into two discrete peaks centred at 2.7 and $4.5 \mathrm{~nm}$ (Fig. 5f). The larger peak value is only slightly smaller than the expected height of $5.0 \mathrm{~nm}$ for the NCP and also agrees with our previous height measurements under the same scanning conditions (Bussiek et al. 2005). Seventy-four percent of the small $2.7 \mathrm{~nm}$ particles were found at DNA termini (an example is given in Fig. 5e). The sample was divided into two subsets, one containing the particles with less than $3.0 \mathrm{~nm}$, the other those above $3.0 \mathrm{~nm}$ height. The wrapped DNA lengths $\left(L_{\mathrm{ncp}}\right)$ of both subsets were determined after contour length measurements of the mononucleosomal fragments: the lengths of free DNA outside the nucleosomes were estimated according to Eq. (1). These lengths were converted to base pairs, taking into account that measured DNA lengths on AFM images can deviate slightly from the expected length of $0.34 \mathrm{~nm} / \mathrm{bp}$ on PL-mica (Bussiek et al. 2003). A measurement of uncomplexed 172- $\alpha 2$ DNAs, present in the same sample preparation, gave a mean contour length of $134.4 \pm 4.3$ (SD) $\mathrm{nm}$ or $0.32 \mathrm{~nm} / \mathrm{bp}$. For each individual complex, the free DNA length in base pairs was subtracted from the total fragment length of 415 bp to obtain $L_{\text {ncp. }}$. The small and the regular particles can be as well discriminated in terms of this length, since both samples yielded two distinct distributions of $L_{\text {ncp }}$ (Fig. $5 \mathrm{~g}$ ). The peak centres of these distributions were at $119 \pm 28$ (SD) bp for the larger particles ( $\geq 3.0 \mathrm{~nm}$ height) and only $84 \pm 37$ (SD) bp for the smaller ones (<3.0 nm height) $(P<0.0001)$. Importantly, the length of $119 \mathrm{bp}$ is in close agreement with the 123 bp determined for the 172- $\alpha 7$ and the 208-6 nucleosome arrays under the same salt conditions. This also provides some evidence that drying the arrays did not extensively distort the nucleosome conformation which we attribute to the irreversible trapping of the DNA to the PL-mica so that the nucleosomes become "fixed" after the binding to the surface.

Nucleosomal positions along the $172-\alpha 2$ AS dimer DNA were determined exclusively for mononucleosomes with particle heights larger than $3.0 \mathrm{~nm}$. Positions were quantified as the ratio $r$, i.e. the distance from the nucleosomal centre to the nearest DNA terminus divided by the total mononucleosomal fragment length (Kepert et al. 2003). The distribution of $r$ shown in Fig. 6 clearly reveals that nucleosomes are preferably localised around the fragment centre, indicated by a defined peak at $r=0.4-0.5$. An $r$-value of $0.5 \pm 0.05$ corresponds to a nucleosomal frame 

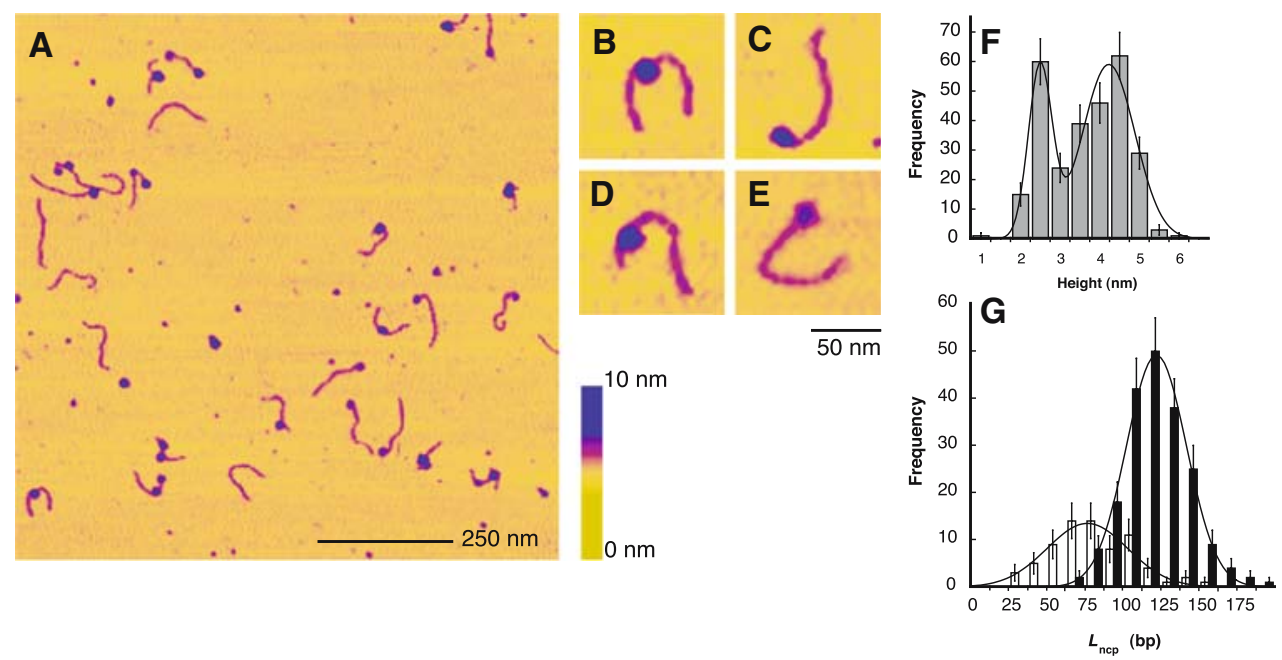

Fig. 5 Atomic force microscopy imaging in liquid of mononucleosomes reconstituted with 172- $\alpha 2$ DNA. Scanning conditions were $10 \mathrm{mM}$ Hepes-NaOH, pH 8.0, $10 \mathrm{mM} \mathrm{NaCl}$. a Overview scan of a sample reconstituted at a nominal octamer to DNA weight ratio of 0.5. b-e Zooms of individual mononucleosomes. $\mathbf{f}$ Height distribution of nucleosomal particles. Frequencies were fitted with the sum of two Gaussian functions to derive mean height values (see text).

whose centre is located next to the EcoRI cleavage site at sequence position $37( \pm 20)$ bp (see Fig. 1). Thus, taking account the error of $r$, the AFM measurement reveals the same prevailing binding site that was estimated by EcoRI cleavage (nucleosomal centre about 10 bp away from the

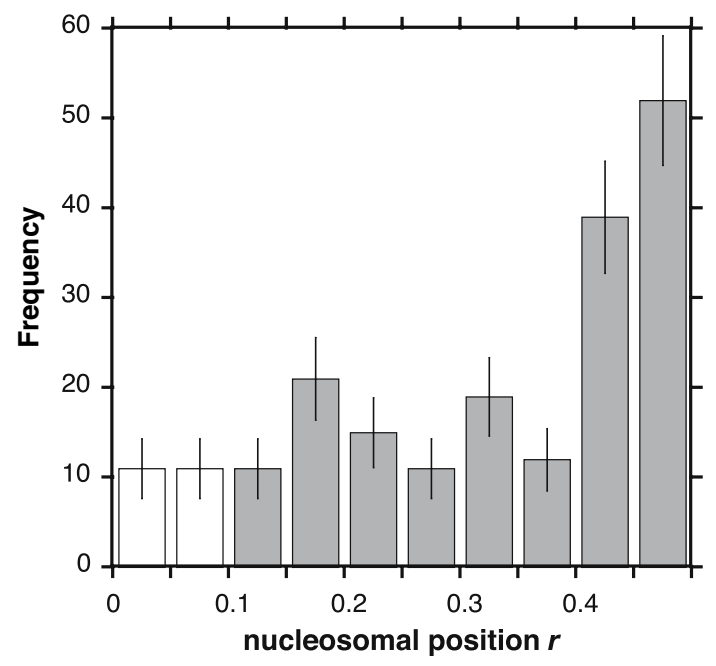

Fig. 6 Positioning of nucleosomes along 172- $\alpha 2$ DNA. Different nucleosomal positions near the termini that would correspond to $r$-values between 0 and 0.1 could not be distinguished due to the resolution limit. Therefore, the occurrences of $r$-values equal to 0 were evenly distributed over the first two bins (white bars). The error of $r$ in the peak range approximately equals the bin size, since an error $\Delta r \sim 10 \%$ could be expected based on the measured standard deviation of the total mononucleosomal fragment length $( \pm 7.7 \%)$ g Distribution of wrapped nucleosomal DNA lengths $L_{\mathrm{ncp}}$, analysed separately for particles $<3.0 \mathrm{~nm}$ height (white bars) and $\geq 3.0 \mathrm{~nm}$ height (black bars). Mean values and standard deviation of $L_{\mathrm{ncp}}$ were calculated by fitting Gaussian functions to both datasets (see text). For comparison with Fig. 3, note that measured height values are larger in liquid than in air, presumably due to reduced sample-probe interactions

EcoRI site, see above). The remaining nucleosomes (55\%) were rather evenly distributed over the eight other bins (with some additional weak minor preferences) and there was no preference for regular size nucleosomes to be aligned with DNA termini.

\section{Discussion}

Nucleosomal positioning along repetitive AGM AS

We reconstituted nucleosomes with dimers (172- $\alpha 2)$ and heptamers (172- $\alpha 7)$ of repetitive AGM AS by the salt dialysis method. Enzymatic digestions and atomic force microscopy were used to characterise the nucleosomal organisation along this DNA. The results demonstrate the formation of a well-defined (sequence-dependent) nucleosomal arrangement, essentially similar to the 5S rDNA model system. In the AGM AS arrays, however, the variation in the separation between neighbouring nucleosomes was rather large, and evenly spaced nucleosomes occurred only to a limited extent.

A partial digestion of $172-\alpha 7$ nucleosome arrays with micrococcal nuclease (MNase) yielded a fragment ladder documenting the presence of phased rather than random nucleosome positions on 172- $\alpha 7$ DNA. This result is very similar to MNase digestions of AGM AS chromatin in cell nuclei (Musich et al. 1982). Core particle length DNA derived from 172- $\alpha 7$ nucleosome arrays was further digested with EcoRI. This produced discrete secondary 
fragments and thus verified the occupation of preferred nucleosomal binding sites. One of these sites was prevailing with a position consistent with published results (Musich et al. 1982; Neubauer et al. 1986; Zhang et al. 1983). One additional minor frame could be detected, which may explain a suggested biphasic cleavage pattern produced by the MNase. Musich et al. (1982) concluded that the MNase digestion of AGM AS chromatin depends on the nucleosomal organisation which includes the possibility that a sequence-specific cleavage coincidentally occurs in the internucleosomal linkers between nucleosomes bound at the mostly preferred site (Horz et al. 1983; Musich et al. 1982). This may lead to a selective representation of the fraction of phased nucleosomes and obscure deviations thereof. Although this source of error must be taken into account, the similar results for cellular AGM AS chromatin and reconstituted nucleosome arrays indicate similar properties, i.e. at least fractions of the nucleosomes are regularly arranged.

Atomic force microscopy imaging was used to detect the spacing between all neighbouring nucleosome pairs in a sample. This circumvents possible artefacts caused by enzymatic analyses, although the precision of localising the nucleosomes is lower. Centre-to-centre $(c c)$ internucleosomal distances measured by AFM imaging not only depend on nucleosomal positions relative to the DNA sequence but also on internucleosome interactions (which should be reduced under the low salt conditions in our measurements) and variations in the amount of wrapped nucleosomal DNA. An example is given by increasing $c c$-distances due to a release of nucleosomal DNA from the histone surface after removal of linker histones (Zlatanova et al. 1998). Especially the wrapped DNA length is susceptible to experimental conditions: a prevailing $c c$-distance of $\sim 20 \mathrm{~nm}$ was previously measured in 5S positioning sequence repeat (208-12) nucleosome arrays that were adsorbed to naked mica in the presence of $\mathrm{Mg}^{2+}$ (Nikova et al. 2004). These measurements used arrays not fixed with glutaraldehyde. Fixed 208-12 nucleosome arrays adsorbed to either spermidine- or AP-mica under low salt conditions gave $c c$-distances of $\sim 30 \mathrm{~nm}$ (Mechelli et al. 2004; Yodh et al. 2002). Our measurements in low salt conditions using PL-mica and unfixed material yielded a larger $c c$-distance for the 208-6 nucleosome arrays of 35$47 \mathrm{~nm}$. An estimation of the wrapped DNA length at our conditions yielded $\sim 120 \mathrm{bp}$, corresponding to $\sim 1.4$ superhelical turns around the octamer [full core particle length of 147 bp equals 1.7 turns (Davey et al. 2002; Furrer et al. 1995; Hamiche et al. 1996; Luger et al. 1997)]. The expected $c c$-distance for a nucleosomal repeat length of $208 \mathrm{bp}$ on the basis of the $120 \mathrm{bp}$ of wrapped DNA is $\sim 40 \mathrm{~nm}$, in agreement with the results obtained for the 2086 arrays. The expected distances for additional unoccupied repeats matched the position of recurring peaks in the $c c$-distance distributions measured for the 208-6 and the 172- $\alpha 7$ nucleosome arrays.

Essentially, the shapes of the $c c$-distance distributions for the 208-6 and the 172- $\alpha 7$ DNA shown in Fig. $4 \mathrm{~b}$ and $c$ are very similar, which points to a sequence dependence of the nucleosomal organisation along repetitive AGM AS similarly strong as on the $5 \mathrm{~S}$ sequence. However, in the case of the 172- $\alpha 7$ DNA, the initial peak of the $c c$-distance distribution, corresponding to the distance between a nucleosome and its nearest neighbour located on an adjacent repeat, is significantly broader and shifted to larger distances than could be expected for evenly spaced nucleosomes. The distributions still show a high frequency of distances up to $\sim 60 \mathrm{~nm}$ (corresponding to $\sim 145 \mathrm{bp}$ of linker DNA length), almost three times the linker length expected for a uniform phasing with the AGM AS repeats (18 $\mathrm{nm}$ or $52 \mathrm{bp}$, based on the $120 \mathrm{bp}$ of wrapped nucleosomal DNA). Thus, many nucleosomal positions were separated by up to about $1.5 \mathrm{AS}$ monomer lengths. The loading with nucleosomes did not significantly affect this result (compare Fig. 4a, b). 208-6 nucleosome arrays showed the expected distance distribution for nucleosomes predominantly bound at and near the main phase, showing that the broad initial peak seen in the 172- $\alpha 7$ arrays was not simply due to a limited measurement accuracy or inappropriate reconstitution or sample preparation conditions. Thus, a strictly regular nucleosomal arrangement occurred in the AGM AS only to limited extent and variation in possible internucleosomal spacings is rather large. We conclude that the number of alternative sites is nevertheless small, because the gelelectrophoresis showed two identifiable sites and the initial peak in the $c c$-distance distribution is sharply separated from the first recurring peak.

The mapping of nucleosomal positions by AFM imaging of the $415 \mathrm{bp}$ long 172- $\alpha 2$ mononucleosomes was fully consistent with the occurrence of the known major positioning site, in addition to the identification of the same site in the nucleosome arrays by EcoRI digestion. The frequency of nucleosomes found at or near this major frame $(45 \%)$ is lower than found by in vitro reconstitutions using a 250 bp AGM AS fragment (80\%) (Neubauer et al. 1986), which is explained by the length difference of the DNA fragments used. The frequency is closer to the observed occupancy of the major site in vivo (35\%) (Zhang et al. 1983) and also closer to that estimated by gelelectrophoretic quantification of the EcoRI digested mononucleosomal DNA derived from the 172- $\alpha 7$ arrays (upper limit of $50 \%$ ). The DNA fragment used here for the reconstitution of the mononucleosomes is the minimal length that contains the AGM AS sequence uninterrupted, such that all possible binding sites are present. However, when 
comparing the positioning observed here and in vivo, it must be taken into account that a few possible binding sites occur twice in the $172-\alpha 2$ (i.e. those which do not incorporate the EcoRI site) and that some additional sites are present that include vector DNA at the ends (33 and $37 \mathrm{bp}$, respectively). In general, positioning on small DNA fragments can be influenced by a preference for DNA-ends (which was not observed here, as shown in Fig. 6) and the length of the fragment used.

A surprising feature was a bimodal height distribution of mononucleosomal particles reconstituted with the 172- $\alpha 2$ DNA. The two height values were correlated with distinguishable wrapped DNA lengths. Nikova et al. (2004) obtained a very similar height distribution by AFM imaging of subsaturated 208-12 nucleosome arrays and presented evidence that particles with only $\sim 75$ bp of wrapped DNA and $\sim 2.5 \mathrm{~nm}$ height correspond to sub-nucleosomal particles that do not contain the full histone octamer. In the present study, small particles of similar dimension (wrapped length of $85 \mathrm{bp}$ and $2.7 \mathrm{~nm}$ height) were predominantly located at the termini of the $172-\alpha 2$ DNA (74\%). In contrast, no preferential binding to termini was found for nucleosomes of regular size $(4.5 \mathrm{~nm}$ height). We may speculate that this high frequency of the small particles at the fragment termini is as well associated with sequence properties of the AGM AS. In our case, the AS sequence starts and ends at the EcoRI site, which is located near the centre of the major binding site. This implies that the end regions of the fragment contain only parts of this binding site. Assuming that multiple DNA-histone interaction along the entire core particle length are responsible for the binding specificity (Neubauer et al. 1986), it may be possible that histones interact preferentially even with parts of the major site. Then, a complete nucleosome assembly could be impeded at the fragment termini due to a lack of sufficient amounts of DNA in the required direction, leading to smaller particles.

\section{Relevance of AGM AS for centromeric chromatin structures}

The kinetochore protein CENP-B is supposed to be involved in nucleosomal positioning in human AS (Tanaka et al. 2005). A structural motive consisting of the AS monomer, nucleosomal positioning and founding kinetochore protein binding may be involved in specifying the location of centromeric function. CENP-B is not present at the centromeres of AGM and therefore AS arrays of this species might compensate for the absence of CENP-B by influencing nucleosomal positioning by their sequence alone (Goldberg et al. 1996). We show here that the AGM AS indeed has a profound influence on the arrangement of oligonucleosomal arrays. This arrangement has similarities to the $5 \mathrm{~S}$ rDNA model system, although multiple positioning leads to larger variation of internucleosome spacings in the AGM AS. The spacing of nucleosomes is expected to contribute to the higher order structuring of chromatin, therefore it is of interest, for example with regard to the design of particular synthetic chromatin structures, to what extent the in vitro produced nucleosomal arrangement along a DNA template resembles that occurring in vivo. Our MNase digestions suggested a high fraction of evenly spaced nucleosomes, similar to digestions of AGM AS in cell nuclei (Musich et al. 1982) whereas the AFM results are consistent with more detailed studies in vivo which demonstrated the occupation of different minor binding sites in addition to the major site (Zhang et al. 1983). The occupancy of the major binding site was in the range of $45-50 \%$ in our analysis which is also in a quite good agreement with the result of the cited in vivo work (35\%). This may further support that the AS sequence contributes significantly to the determination of AS chromatin and probably to centromeric chromatin structures. The DNA constructs built in this study will give the opportunity to investigate further the structure and biophysical properties of AS chromatin and centromeric chromatin. For example, it will be important to compare the nucleosomal arrangement on repetitive AGM and human AS (with and without CENP-B boxes). Nucleosomal phasing also occurs on human AS sequences (Tanaka et al. 2005; Yoda et al. 1998) and a preferred positioning site found in immunoprecipitated human centromeric AS indeed corresponded to that occurring in AGM AS (Vafa and Sullivan 1997).

Acknowledgements We are grateful to Nathalie Schwarz, Maria Mildenberger and Claus Rodemer for technical assistance. Also we thank Peter Becker for the vector containing the repetitive 5S rDNA and Michael Poirier for helpful proofreading and comments on the manuscript.

\section{References}

Alexandrov I, Kazakov A, Tumeneva I, Shepelev V, Yurov Y (2001) Alpha-satellite DNA of primates: old and new families. Chromosoma 110:253-266

Allen MJ, Dong XF, O’Neill TE, Yau P, Kowalczykowski SC, Gatewood J, Balhorn R, Bradbury EM (1993) Atomic force microscope measurements of nucleosome cores assembled along defined DNA sequences. Biochemistry 32:8390-8396

Amor DJ, Choo KH (2002) Neocentromeres: role in human disease, evolution, and centromere study. Am J Hum Genet 71:695-714

Blank TA, Becker PB (1996) The effect of nucleosome phasing sequences and DNA topology on nucleosome spacing. J Mol Biol 260:1-8

Brown FL, Musich PR, Maio JJ (1979) The repetitive sequence structure of component alpha DNA and its relationship to the nucleosomes of the African green monkey. J Mol Biol 131:777799 
Bussiek M, Mucke N, Langowski J (2003) Polylysine-coated mica can be used to observe systematic changes in the supercoiled DNA conformation by scanning force microscopy in solution. Nucleic Acids Res 31:e137

Bussiek M, Toth K, Brun N, Langowski J (2005) DNA-loop formation on nucleosomes shown by in situ scanning force microscopy of supercoiled DNA. J Mol Biol 345:695-706

Carruthers LM, Bednar J, Woodcock CL, Hansen JC (1998) Linker histones stabilize the intrinsic salt-dependent folding of nucleosomal arrays: mechanistic ramifications for higher-order chromatin folding. Biochemistry 37:14776-14787

Davey CA, Sargent DF, Luger K, Maeder AW, Richmond TJ (2002) Solvent mediated interactions in the structure of the nucleosome core particle at 1.9 a resolution. J Mol Biol 319:1097-1113

Fittler F, Zachau HG (1979) Subunit structure of alpha-satellite DNA containing chromatin from African green monkey cells. Nucleic Acids Res 7:1-13

Fitzgerald DJ, Dryden GL, Bronson EC, Williams JS, Anderson JN (1994) Conserved patterns of bending in satellite and nucleosome positioning DNA. J Biol Chem 269:21303-21314

Furrer P, Bednar J, Dubochet J, Hamiche A, Prunell A (1995) DNA at the entry-exit of the nucleosome observed by cryoelectron microscopy. J Struct Biol 114:177-183

Gilbert N, Allan J (2001) Distinctive higher-order chromatin structure at mammalian centromeres. Proc Natl Acad Sci USA 98:11949_ 11954

Goldberg IG, Sawhney H, Pluta AF, Warburton PE, Earnshaw WC (1996) Surprising deficiency of CENP-B binding sites in African green monkey alpha-satellite DNA: implications for CENP-B function at centromeres. Mol Cell Biol 16:5156-5168

Gruss P, Sauer G (1975) Repetitive primate DNA containing the recognition sequences for two restriction endonucleases which generate cohesive ends. FEBS Lett 60:85-88

Hamiche A, Schultz P, Ramakrishnan V, Oudet P, Prunell A (1996) Linker histone-dependent DNA structure in linear mononucleosomes. J Mol Biol 257:30-42

Horz W, Fittler F, Zachau HG (1983) Sequence specific cleavage of African green monkey alpha-satellite DNA by micrococcal nuclease. Nucleic Acids Res 11:4275-4285

Kepert JF, Toth KF, Caudron M, Mucke N, Langowski J, Rippe K (2003) Conformation of reconstituted mononucleosomes and effect of linker histone $\mathrm{H} 1$ binding studied by scanning force microscopy. Biophys J 85:4012-4022

Linxweller W, Horz W (1985) Reconstitution experiments show that sequence-specific histone-DNA interactions are the basis for nucleosome phasing on mouse satellite DNA. Cell 42:281-290

Luger K, Mader AW, Richmond RK, Sargent DF, Richmond TJ (1997) Crystal structure of the nucleosome core particle at $2.8 \mathrm{~A}$ resolution. Nature 389:251-260

Luger K, Rechsteiner TJ, Richmond TJ (1999) Preparation of nucleosome core particle from recombinant histones. Methods Enzymol 304:3-19

Masumoto H, Masukata H, Muro Y, Nozaki N, Okazaki T (1989) A human centromere antigen (CENP-B) interacts with a short specific sequence in alphoid DNA, a human centromeric satellite. J Cell Biol 109:1963-1973

Mechelli R, Anselmi C, Cacchione S, De Santis P, Savino M (2004) Organization of telomeric nucleosomes: atomic force microscopy imaging and theoretical modeling. FEBS Lett 566:131-135
Meersseman G, Pennings S, Bradbury EM (1991) Chromatosome positioning on assembled long chromatin. Linker histones affect nucleosome placement on 5 S rDNA. J Mol Biol 220:89-100

Musich PR, Brown FL, Maio JJ (1982) Nucleosome phasing and micrococcal nuclease cleavage of African green monkey component alpha DNA. Proc Natl Acad Sci USA 79:118-122

Neubauer B, Linxweiler W, Horz W (1986) DNA engineering shows that nucleosome phasing on the African green monkey alphasatellite is the result of multiple additive histone-DNA interactions. J Mol Biol 190:639-645

Nikova DN, Pope LH, Bennink ML, van Leijenhorst-Groener KA, van der Werf K, Greve J (2004) Unexpected binding motifs for subnucleosomal particles revealed by atomic force microscopy. Biophys J 87:4135-4145

Rosenberg H, Singer M, Rosenberg M (1978) Highly reiterated sequences of SIMIANSIMIANSIMIANSIMIANSIMIAN. Science 200:394-402

Schueler MG, Higgins AW, Rudd MK, Gustashaw K, Willard HF (2001) Genomic and genetic definition of a functional human centromere. Science 294:109-115

Segal E, Fondufe-Mittendorf Y, Chen L, Thastrom A, Field Y, Moore IK, Wang JP, Widom J (2006) A genomic code for nucleosome positioning. Nature 442:772-778

Simpson RT, Thoma F, Brubaker JM (1985) Chromatin reconstituted from tandemly repeated cloned DNA fragments and core histones: a model system for study of higher order structure. Cell 42:799-808

Smith MR, Lieberman MW (1984) Nucleosome arrangement in alpha-satellite chromatin of African green monkey cells. Nucleic Acids Res 12:6493-6510

Tanaka Y, Tachiwana H, Yoda $\mathrm{K}$, Masumoto $\mathrm{H}$, Okazaki T, Kurumizaka H, Yokoyama S (2005) Human centromere protein $\mathrm{B}$ induces translational positioning of nucleosomes on alphasatellite sequences. J Biol Chem 280:41609-41618

Vafa O, Sullivan KF (1997) Chromatin containing CENP-A and $\alpha$ satellite DNA is a major component of the inner kinetochore plate. Curr Biol 7:897-900

Willard HF (1998) Centromeres: the missing link in the development of human artificial chromosomes. Curr Opin Genet Dev 8:219 225

Wu KC, Strauss F, Varshavsky A (1983) Nucleosome arrangement in green monkey alpha-satellite chromatin. Superimposition of non-random and apparently random patterns. J Mol Biol 170:93117

Yoda K, Ando S, Okuda A, Kikuchi A, Okazaki T (1998) In vitro assembly of the CENP-B/alpha-satellite DNA/core histone complex: CENP-B causes nucleosome positioning. Genes Cells 3:533-548

Yodh JG, Woodbury N, Shlyakhtenko LS, Lyubchenko YL, Lohr D (2002) Mapping nucleosome locations on the 208-12 by AFM provides clear evidence for cooperativity in array occupation. Biochemistry 41:3565-3574

Zhang XY, Fittler F, Horz W (1983) Eight different highly specific nucleosome phases on alpha-satellite DNA in the African green monkey. Nucleic Acids Res 11:4287-4306

Zlatanova J, Leuba SH, van Holde K (1998) Chromatin fiber structure: morphology, molecular determinants, structural transitions. Biophys J 74:2554-2566 